\title{
Heat transfer mechanisms in bubbly Rayleigh-Bénard convection
}

\author{
Paolo Oresta, ${ }^{1}$ Roberto Verzicco, ${ }^{2}$ Detlef Lohse, ${ }^{1}$ and Andrea Prosperetti ${ }^{1,3}$ \\ ${ }^{1}$ Physics of Fluids Group, Department of Science and Technology, J. M. Burgers Centre for Fluid Dynamics, and Impact Institute, \\ University of Twente, P.O. Box 217, 7500 AE Enschede, The Netherlands \\ ${ }^{2}$ Department of Mechanical Engineering, University of Rome "Tor Vergata," Via del Politecnico 1, 00133 Rome, Italy \\ ${ }^{3}$ Department of Mechanical Engineering, Johns Hopkins University, Baltimore, Maryland 21218, USA
}

(Received 12 November 2008; published 17 August 2009)

\begin{abstract}
The heat transfer mechanism in Rayleigh-Bénard convection in a liquid with a mean temperature close to its boiling point is studied through numerical simulations with pointlike vapor bubbles, which are allowed to grow or shrink through evaporation and condensation and which act back on the flow both thermally and mechanically. It is shown that the effect of the bubbles is strongly dependent on the ratio of the sensible heat to the latent heat as embodied in the Jakob number Ja. For very small Ja the bubbles stabilize the flow by absorbing heat in the warmer regions and releasing it in the colder regions. With an increase in Ja, the added buoyancy due to the bubble growth destabilizes the flow with respect to single-phase convection and considerably increases the Nusselt number.
\end{abstract}

DOI: 10.1103/PhysRevE.80.026304

PACS number(s): 47.20.Bp, 47.55.-t

\section{INTRODUCTION}

Thermal convection is an omnipresent phenomenon in nature and technology. The idealized version thereof is the Rayleigh-Bénard (RB) convection-a single-phase fluid in a closed container heated from below and cooled from above. A key question is the dependence of the heat transfer rate (as measured by the Nusselt number) for a given temperature difference between the hot bottom and the cold top plates (i.e., Rayleigh number), a given fluid (i.e., Prandtl number), and a given aspect ratio. In the last two decades there has been tremendous progress on this and related questions by experiment, theory, and numerical simulation (see $[1,2]$ for a recent review). Most of the work focused on the RB convection for single-phase flow. Various situations in the process and the energy industries, however, involve liquid convection in the presence of boiling: vapor generators in nuclear and conventional electric power plants, reboilers, distillers, water purification systems, cooling applications and many others.

The effectiveness of boiling as a heat transfer mechanism has been known for centuries and the process has formed the object of a very large number of studies [3]. Most of the focus has been on the process by which the high thermal resistance opposed by the viscothermal layer adjacent to the hot surface is decreased by the vapor bubbles; the two main mechanisms are believed to be microconvection and latent heat transport. Another significant effect of the bubbles, however, is to promote strong convective currents in the liquid, thus helping to remove the heated layer near the hot wall. This aspect of the process forms the object of the present study.

In an actual experiment-see, e.g., the recent measurements of Zhong et al. [4] with ethane close to the critical point - all the processes occur at the same time and it is next to impossible to separately quantify their relative importance. Numerical simulation appears to be a promising tool for this purpose. Ideally, a simulation should be able to resolve individual bubbles and follow their evolution but, with the present capabilities, only so few bubbles can be simulated to this level of detail that it would be very difficult to draw conclusive results [5-8]. Therefore, one has to fall back on point-bubble models in which the interaction of the individual bubbles with the surrounding liquid is parametrized. This approach has proven valuable in the study of turbulence in particle-laden flows (see, e.g., [9-11]), in liquids with gas-rather than vapor-bubbles [12-14] and for TaylorCouette flow with microbubbles inducing a drag reduction [15].

Many important physical mechanisms have been elucidated by these means and one may therefore hope that similar insights might be achieved by extending this line of research accounting for phase change processes, and the accompanying bubble growth and collapse, in a similar way. Thus, to the fluid mechanics bubble-liquid interaction model used in our earlier work [13,14], we add here models for the heat transfer and phase change between the bubbles and the surrounding liquid along the lines of Refs. [16,17].

The standard single-phase RB convection under the Boussinesq approximation is controlled by the Rayleigh number

$$
\mathrm{Ra}=\frac{g \beta\left(T_{h}-T_{c}\right) H^{3}}{\nu \kappa},
$$

where $T_{h}$ and $T_{c}$ are the temperatures of the hot (bottom) and the cold (top) plates, respectively; $H$ is the height of the convection cell; $g$ is the gravitational acceleration; $\kappa$ is the thermal diffusivity of the liquid; $\nu$ is its kinematic viscosity; and $\beta$ is the isobaric thermal expansion coefficient. The Prandtl number is defined as

$$
\operatorname{Pr}=\frac{\nu}{\kappa} .
$$

In this paper we consider convection for which, without bubbles, $\mathrm{Ra}=2 \times 10^{5}$ and $\operatorname{Pr}=1.75$ (water at $100{ }^{\circ} \mathrm{C}$ ); the cell is cylindrical and the aspect ratio (defined as the ration of the diameter to the height) is $1 / 2$. With these parameter 

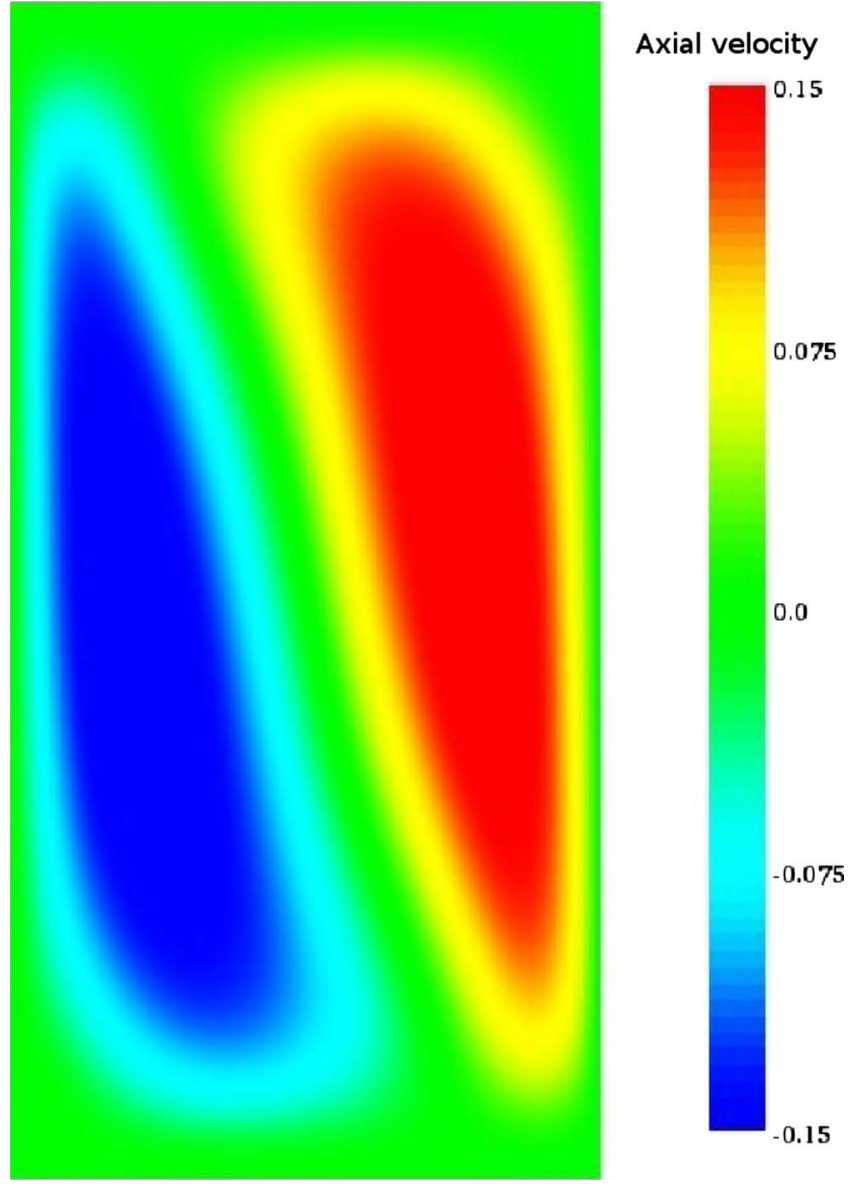

FIG. 1. (Color online) Vertical velocity in the plane of symmetry of the full cylinder in the absence of bubbles; $\mathrm{Ra}=2 \times 10^{5}, \mathrm{Pr}$ $=1.75, \mathrm{Nu}=4.75$. As throughout the paper, the velocity is made dimensionless by using the free-fall velocity $\left[\beta g H\left(T_{h}-T_{c}\right)\right]^{1 / 2}$.

values, in the absence of bubbles, there is a convection roll with fluid rising along one side of the cell and descending along the opposite side (see Fig. 1); the Nusselt number has the value of 4.75 .

Vapor bubbles introduce a crucial new parameter, the Jakob number,

$$
\mathrm{Ja}=\frac{\rho c_{p}\left(T_{h}-T_{\text {sat }}\right)}{\rho_{V} L}
$$

in which $L$ is the latent heat; $\rho_{V}$ and $\rho$ are the vapor and the liquid densities, respectively; $c_{p}$ is the liquid specific heat; and $T_{\text {sat }}$ is the saturation temperature of the liquid. With the parameter values used in this study, hydrostatic pressure variations are not sufficient to cause a significant change in $T_{\text {sat }}$, which therefore is taken as a constant equal to the average of the hot and the cold plate temperatures. Physically, Ja represents the ratio of the sensible heat to the latent heat. A very small Jakob number may be thought of as a very large value of the latent heat, which will tend to limit the volume change of the bubbles due to evaporation or condensation.

For $\mathrm{Ja}=0$, the latent heat is effectively infinite and bubbles cannot grow or shrink: they maintain their initial diameter at nucleation, which we take to be $2 R_{b 0}=25 \mu \mathrm{m}$.
Another control parameter in our model is the total number $N_{b}$ of bubbles in the cylinder. Although in real systems this number will fluctuate in time somewhat, here, we take it as constant: whenever a bubble reaches the top of the cylinder and is removed, a new bubble of the standard initial size (25 $\mu \mathrm{m})$ is nucleated at the bottom plate at some random position.

\section{MODEL}

We study the problem in the standard Boussinesq approximation augmented by the momentum and energy effects of the bubbles, treated as points. When the volume occupied by the bubbles is very small, the liquid continuity equation retains the standard incompressible form

$$
\boldsymbol{\nabla} \cdot \mathbf{u}=0
$$

in which $\mathbf{u}$ is the liquid velocity field. The momentum equations is

$$
\rho \frac{D \mathbf{u}}{D t}=-\nabla p+\mu \nabla^{2} \mathbf{u}+\beta \rho\left(T-T_{s a t}\right) \mathbf{g}+\sum_{i} \mathbf{f}_{i} \delta\left(\mathbf{x}-\mathbf{x}_{i}\right),
$$

where $D / D t$ is the convective derivative, $p$ and $T$ are the pressure and the temperature, and $\mu=\nu \rho$ is the dynamic viscosity. The effect of the bubbles has been approximated by modeling them as pointlike sources of momentum. The approximations involved in the use of Eqs. (4) and (5) at small bubble volume fractions are standard and amply discussed in the literature, to which the reader is referred for details (see, e.g., [14]).

The position of the center of the $n$th bubble is denoted by $\mathbf{x}_{i}$ and the force $\mathbf{f}_{i}$ that it applies on the liquid is modeled as (see, e.g., $[18,14]$ )

$$
\mathbf{f}_{i}=\frac{4}{3} \pi R_{b i}^{3} \rho\left(\left.\frac{D \mathbf{u}}{D t}\right|_{\mathbf{x}_{i}}-\mathbf{g}\right)
$$

in which $R_{b i}$ is the radius of the $i$ th bubble and the liquid acceleration is evaluated at the position of the bubble. A similar term multiplied by the vapor, rather than the liquid, density is very small and has been neglected here.

The liquid energy equation takes the form (Appendix A)

$$
\rho c_{p} \frac{D T}{D t}=k \nabla^{2} T+\sum_{i} Q_{i} \delta\left(\mathbf{x}-\mathbf{x}_{i}\right),
$$

where $k=\kappa \rho c_{p}$ is the liquid thermal conductivity and $Q_{i}$ is the energy source or sink due to phase change of the $i$ th bubble. We model the thermal exchange between the $i$ th bubble and the liquid by means of a heat transfer coefficient $h_{b i}$ and write

$$
Q_{i}=4 \pi R_{b i}^{2} h_{b i}\left(T_{s a t}-T_{i}\right),
$$

where $T_{i}=T\left(\mathbf{x}_{i}, t\right)$ is the liquid temperature evaluated at the position of the center of the $i$ th bubble. In writing this relation we have used the fact that, for moderate temperature differences, phase change is slow and the bubble surface 
remains essentially at saturated conditions (see, e.g., [19]).

Expressions (6) and (8) and the use of point sources of momentum in Eq. (5) and of energy in Eq. (7) assume that the bubbles interact only through the average fields but not directly, which is a reasonable approximation at the vapor volume fractions considered here (see, e.g., $[13,14]$ ).

Part of the system energy is carried by the bubble phase. If $H_{b}$ denotes the enthalpy of a single bubble, $n$ denotes the bubble number density, and $\mathbf{v}$ denotes the bubble velocity, the conservation of this component of the system energy is expressed by (Appendix A)

$$
\frac{\partial}{\partial t}\left(n H_{b}\right)+\nabla \cdot\left(n H_{b} \mathbf{v}\right)=-\sum_{i} Q_{i} \delta\left(\mathbf{x}-\mathbf{x}_{i}\right) .
$$

Adding Eqs. (7) and (9) gives an equation for the balance of the total system energy, namely,

$$
\begin{aligned}
& \left.\frac{\partial}{\partial t}\left[\rho c_{p}\left(T-T_{s a t}\right)+n H_{b}\right]+\nabla \cdot\left[\rho c_{p}\left(T-T_{s a t}\right) \mathbf{u}+n H_{b} \mathbf{v}\right)\right] \\
& \quad=k \nabla^{2} T
\end{aligned}
$$

With the neglect of the vapor mass, the equation of motion for each bubble balances added mass, drag, lift, and buoyancy,

$$
\begin{aligned}
C_{A} \rho & {\left[\frac{4}{3} \pi R_{b}^{3}\left(\frac{D \mathbf{u}}{D t}-\frac{d \mathbf{v}}{d t}\right)+(\mathbf{u}-\mathbf{v}) \frac{d}{d t}\left(\frac{4}{3} \pi R_{b}^{3}\right)\right] } \\
& -\frac{1}{2} \pi C_{D} \rho R_{b}^{2}|\mathbf{v}-\mathbf{u}|(\mathbf{v}-\mathbf{u})+\frac{4}{3} \pi R_{b}^{3} \rho \frac{D \mathbf{u}}{D t} \\
& +C_{L_{3}} \frac{4}{3} \pi R_{b}^{3} \rho(\nabla \times \mathbf{u}) \times(\mathbf{v}-\mathbf{u})-\frac{4}{3} \pi R_{b}^{3} \rho \mathbf{g}=0
\end{aligned}
$$

in which $C_{A}, C_{L}$, and $C_{D}$ are the added mass, lift, and drag coefficients, respectively. The uncertainty with which many of the terms of this equation are known is well appreciated in the literature (see, e.g., [20] or our own work [21]). Moreover, due to the interaction with the wake, there might be history forces which have been neglected in Eq. (11) [22-24]. Nevertheless, as written, the equation captures the basic effects of drag, buoyancy, and added mass which dominate the bubble-liquid interaction. After some rearrangement, the equation becomes

$$
\begin{aligned}
C_{A} \frac{d \mathbf{v}}{d t}= & \left(1+C_{A}\right) \frac{D \mathbf{u}}{D t}-\frac{3 C_{A}}{R_{b}}(\mathbf{v}-\mathbf{u}) \frac{d R_{b}}{d t} \\
& -\frac{3}{8} \frac{C_{D}}{R_{b}}|\mathbf{v}-\mathbf{u}|(\mathbf{v}-\mathbf{u})-\mathbf{g}+C_{L}(\nabla \times \mathbf{u}) \times(\mathbf{v}-\mathbf{u}) .
\end{aligned}
$$

The bubble radius $R_{b}$ is calculated by balancing the latent heat associated with evaporation or condensation with the heat exchanged with the liquid

$$
L \frac{d}{d t}\left(\frac{4}{3} \pi R_{b}^{3} \rho_{V}\right)=-Q=4 \pi R_{b}^{2} h_{b}\left(T-T_{\text {sat }}\right) .
$$

Since the bubble is assumed to be at saturation, $\rho_{V}$ is a constant and this equation can be simplified to the form

$$
\frac{d R_{b}}{d t}=\frac{h_{b}}{L \rho_{V}}\left(T-T_{s a t}\right)
$$

in which $\rho_{V}=\rho_{V}\left(T_{\text {sat }}\right)$.

In order to complete the mathematical formulation of the problem, definite choices must be made for several quantities. Since our bubbles are small and therefore will not deform very much, we take $C_{A}=1 / 2$, which is the standard potential-flow value for a sphere (see, e.g., [25]), independent of the Reynolds number and of nonuniformities of the flow [26-29]. The inviscid calculation of [27] gives the same value for the lift coefficient; this value appears to be a reasonable estimate even at low to moderate Reynolds number (see Fig. 17 of [23]). We model the drag coefficient as suggested by $[22,30]$ as follows:

$$
C_{D}=\frac{16}{\operatorname{Re}_{b}}\left[1+\frac{\mathrm{Re}_{b}}{8+\frac{1}{2}\left(\operatorname{Re}_{b}+3.315 \sqrt{\mathrm{Re}_{b}}\right)}\right]
$$

in which $\operatorname{Re}_{b}=2 R_{b}|\mathbf{v}-\mathbf{u}| / \nu$ is the bubble Reynolds number.

We express the heat transfer coefficient $h_{b}$ in terms of a single-bubble Nusselt number

$$
\mathrm{Nu}_{b}=\frac{2 R_{b} h_{b}}{k} .
$$

The dependence of $\mathrm{Nu}_{b}$ on the parameters of the problem is complicated and has been studied by several authors (see, e.g., $[16,17])$. In order to make progress, we are forced to introduce some simplifications. The analysis of [16] shows that, as a function of the Péclet number

$$
\mathrm{Pe}_{b}=\frac{2 R_{b}|\mathbf{v}-\mathbf{u}|}{\kappa},
$$

there are essentially two regimes. At low $\mathrm{Pe}_{b}, \mathrm{Nu}_{b}$ is approximately independent of $\mathrm{Pe}_{b}$ and only depends on the Jakob number (3). We call this value $\mathrm{Nu}_{b, 0}$ The functional relationship $\mathrm{Nu}_{b, 0}(\mathrm{Ja})$ in this regime has been variously parametrized by different authors. Reference [16] proposes a general form

$$
\mathrm{Nu}_{b, 0}=\frac{16}{\pi} \mathrm{Ja} f(\mathrm{Ja}) .
$$

For the function $f(\mathrm{Ja})$, Ref. [31] (corroborated by the more recent results of Ref. [17]) proposes

$$
f(\mathrm{Ja})=\frac{\pi}{8 \mathrm{Ja}}+\frac{\left(6 \pi^{2}\right)^{1 / 3}}{16} \frac{1}{\mathrm{Ja}^{2 / 3}}+\frac{3}{4}
$$

with which Eq. (18) becomes

$$
\mathrm{Nu}_{b, 0}=2+\left(\frac{6 \mathrm{Ja}}{\pi}\right)^{1 / 3}+\frac{12}{\pi} \mathrm{Ja} .
$$

For very large Péclet numbers, heat transfer is dominated by convection and the result is [32]

$$
\mathrm{Nu}_{b, \infty}=2 \sqrt{\frac{\mathrm{Pe}_{b}}{\pi}} .
$$

We combine these two asymptotic forms in a way that smoothly interpolates between them, 


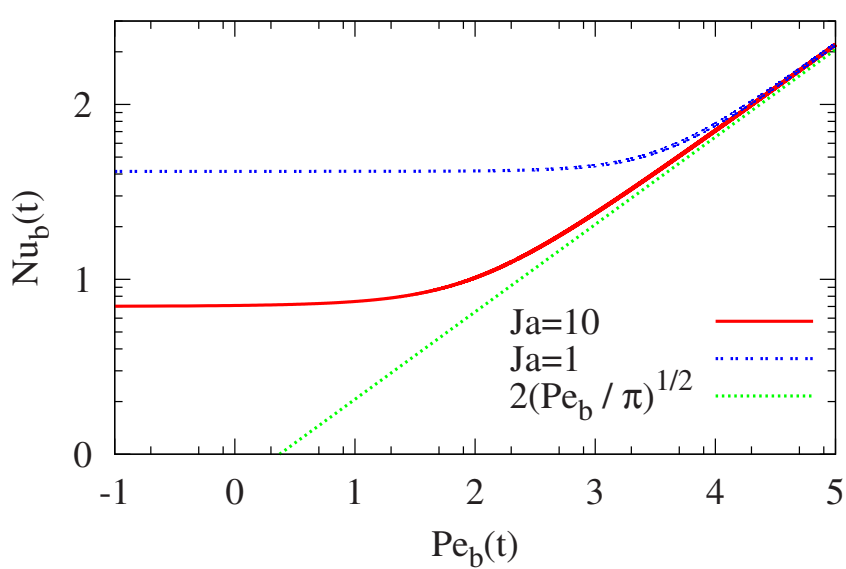

FIG. 2. (Color online) Interpolation (22) for the dependence of the single-bubble Nusselt number on the Péclet number.

$$
\mathrm{Nu}_{b}=\mathrm{Nu}_{b, 0}\left[1+\left(\frac{\mathrm{Pe}_{b}}{\mathrm{Pe}_{c}}\right)^{n / 2}\right]^{1 / n},
$$

where $n \simeq 2.65$ is determined by fitting the results of Refs. $[17,32]$ and the crossover Péclet number $\mathrm{Pe}_{c}$, defined by $\mathrm{Nu}_{b, \infty}=\mathrm{Nu}_{b, 0}$, is $\mathrm{Pe}_{c}=\pi \mathrm{Nu}_{b, 0}^{2} / 4$. Relation (22) is shown as a function of $\mathrm{Pe}_{b}$ for $\mathrm{Ja}=1$ and 10 in Fig. 2. These results can be compared with the corresponding ones presented in Fig. 3 of [16] and are seen to provide an accurate representation of them.

\section{NUSSELT NUMBER}

If the total-energy equation (10) is averaged over time and integrated over the cylinder volume, we find

$$
\left.\left\langle n H_{b} v_{3}-k \partial_{3} T\right\rangle_{A, t}\right|_{z=H}=\left.\left\langle n H_{b} v_{3}-k \partial_{3} T\right\rangle_{A, t}\right|_{z=0},
$$

where the subscript 3 denotes the vertical direction and $\langle\cdots\rangle_{A, t}$ denotes the time and the area averages. In deriving this relation, we have used the no-slip condition for the liq-

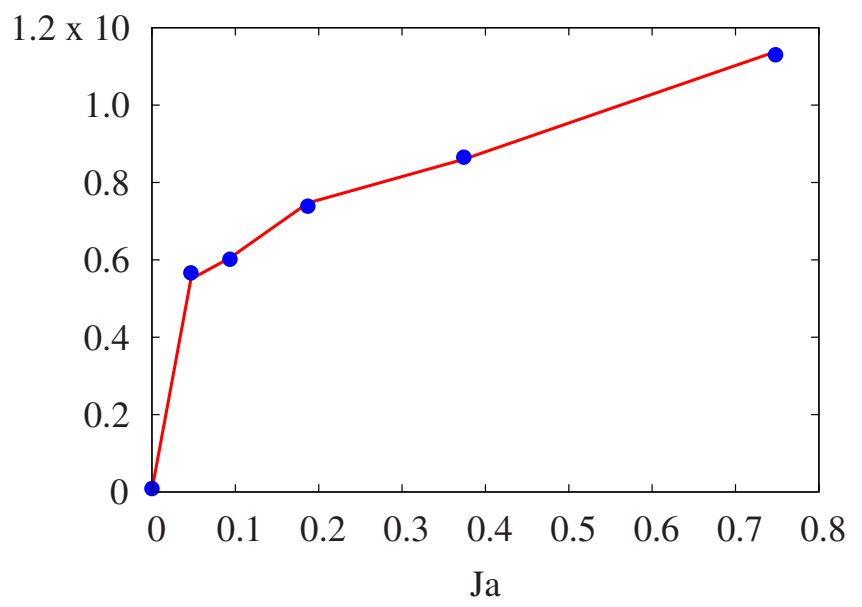

FIG. 3. (Color online) The line shows the numerical results for the left-hand side of Eq. (29) and the points those for the right-hand side. Equality of these two quantities is a stringent of the accuracy of the computation. $N_{b}=5000$. uid phase and the assumed adiabaticity of the lateral walls. A similar treatment of the bubble energy equation (9) gives

$$
\left.\left\langle n H_{b} v_{3}\right\rangle_{A, t}\right|_{z=H}-\left.\left\langle n H_{b} v_{3}\right\rangle_{A, t}\right|_{z=0}=-\frac{1}{\pi R^{2}}\left\langle\sum_{i} Q_{i}\right\rangle_{t},
$$

where $R$ is the radius of the cylinder. The summation in the last term is over all the bubbles contained in the system and the average is over time. Note that, since bubbles are injected and removed at the bottom and the top plates, the bubble velocity will not vanish at $z=0$ and $z=H$.

Using Eq. (24), Eq. (23) can equivalently be written as

$$
-\left.\left\langle k \partial_{3} T\right\rangle_{A, t}\right|_{z=H}+\left.k\left\langle\partial_{3} T\right\rangle_{A, t}\right|_{z=0}=\frac{1}{\pi R^{2}}\left\langle\sum_{i} Q_{i}\right\rangle_{t},
$$

which expresses the obvious fact that any difference between the heat conducted out of the bottom plate and into the top plate is due to the energy stored in the bubbles.

In single-phase natural convection, the conventional definition of the Nusselt numbers $\mathrm{Nu}_{c}$ and $\mathrm{Nu}_{h}$ at the hot and the cold plates is

$$
\mathrm{Nu}_{c, h}=-\left.\frac{H}{T_{h}-T_{c}}\left\langle\partial_{3} T\right\rangle_{A, t}\right|_{z=H, z=0} .
$$

In the single-phase case, this quantity may be considered as a total dimensionless heat flux, but this interpretation would be incorrect here as it disregards the effect of the bubbles. Here, the proper quantity to be regarded as the total dimensionless heat flux would be

$$
N_{c, h}^{*}=\left.\frac{H}{k\left(T_{h}-T_{c}\right)}\left\langle n H_{b} v_{3}-k \partial_{3} T\right\rangle_{A, t}\right|_{z=H, 0}
$$

which, by Eq. (23), satisfies

$$
N_{h}^{*}=N_{c}^{*}
$$

as expected. However, since the point of this paper is to show the impact of the bubbles on what would be considered the heat flux in single-phase convection, it is preferable to present our results in terms of $\mathrm{Nu}_{h, c}$ rather than $N_{h, c}^{*}$.

Definitions (26) lead to

$$
\mathrm{Nu}_{c}-\mathrm{Nu}_{h}=\frac{H}{\pi R^{2} k\left(T_{h}-T_{c}\right)}\left\langle\sum_{i} Q_{i}\right\rangle_{t} .
$$

Separate expressions for $\mathrm{Nu}_{c}$ and $\mathrm{Nu}_{h}$ can be found by using another relation which can be derived by multiplying Eq. (7) by $z-\frac{1}{2} H$ and integrating over the volume of the cylinder with the result

$$
\begin{aligned}
\overline{\mathrm{Nu}} & \equiv \frac{1}{2}\left(\mathrm{Nu}_{c}+\mathrm{Nu}_{h}\right) \\
& =1+\frac{H}{\kappa \Delta}\left\langle u_{3}\left(T-T_{\text {sat }}\right)\right\rangle_{V, t}+\frac{1}{\pi R^{2} k \Delta}\left\langle\sum_{i}\left(z_{i}-\frac{1}{2} H\right) Q_{i}\right\rangle_{t}
\end{aligned}
$$

in which $\langle\cdots\rangle_{V, t}$ denotes time and volume averages and $\Delta$ $=T_{h}-T_{c}$; in the following we refer to $\overline{\mathrm{Nu}}$ as the average Nusselt number. By using this relation and Eq. (29), we have 


$$
\mathrm{Nu}_{h}=1+\frac{H}{\kappa \Delta}\left\langle u_{3}\left(T-T_{s a t}\right)\right\rangle_{V, t}+\frac{1}{\pi R^{2} k \Delta}\left\langle\sum_{i}\left(z_{i}-H\right) Q_{i}\right\rangle_{t}
$$

and

$$
\mathrm{Nu}_{c}=1+\frac{H}{\kappa \Delta}\left\langle u_{3}\left(T-T_{s a t}\right)\right\rangle_{V, t}+\frac{1}{\pi R^{2} k \Delta}\left\langle\sum_{i} z_{i} Q_{i}\right\rangle_{t} .
$$

To estimate the dimensionless total heat fluxes $N_{h, c}^{*}$, we note that, since bubbles are injected with a small velocity and a small radius, at the hot plate the first term in Eq. (27) is negligible compared with the second one, so that

$$
N_{c}^{*}=N_{h}^{*} \simeq \mathrm{Nu}_{h} .
$$

Just as the Nusselt number, the expressions for the kinetic and the thermal dissipations $\epsilon_{u}$ and $\epsilon_{\theta}$ of standard singlephase natural convection are also affected by the bubble contribution to the liquid energy equation. These modified expressions are derived in Appendix B.

\section{NUMERICAL METHODS}

For the liquid phase, we solve the continuity, the momentum, and the energy equations (4), (5), and (7) written in cylindrical coordinates. Once the liquid fields have been calculated, the bubble radius is found from Eq. (14) and the bubble velocity from Eq. (12).

The discretization of the liquid-phase equations is carried out using a staggered second-order-accurate finite difference scheme. The resulting algebraic system is solved by a fractional step method with the advective terms treated explicitly and the viscous terms computed implicitly by an approximate factorization technique (see [33] for details). The Poisson equation that enforces the flow incompressibility is solved by a direct procedure which relies on trigonometric expansions in the azimuthal direction and the FISHPACK package [34] for the radial and the axial directions for which, therefore, a nonuniform mesh distribution can be used. The grid is nonuniform in the radial and the axial directions and clustered toward the boundaries to adequately resolve the viscous and the thermal layers. Most of the simulations were conducted with a grid having $33 \times 25 \times 80$ nodes in the azimuthal, the radial, and the axial directions, respectively. As a check, for a few cases we used a finer grid with $33 \times 40$ $\times 120$ nodes finding results within $1.5 \%$ of those obtained with the coarser grid. To assess the adequacy of the spatial discretization in the vertical direction, we can estimate the thickness $\lambda_{T}$ of the thermal boundary layers as $\Delta /\left(2 \lambda_{T}\right)$ $\sim\left\langle\partial_{3} T\right\rangle$ or, from Eq. (26), $\lambda_{T} \sim H /(2 \mathrm{Nu})$. The largest Nusselt numbers encountered in this work are about 50 and the vertical discretization with 80 nodes places at least six points inside the thermal boundary layer.

The time advancement of the solution has been carried out by a simple third-order Runge-Kutta procedure. Rather than from stability requirements for the calculation of the continuous fields, the most stringent limitation on the time step arises from Eq. (12) for the bubble velocity. The root of the difficulty lies in the very short relaxation time of the dynamics of small bubbles. To deal with this problem, the bubble momentum equation (12) is integrated implicitly by the trapezoidal rule. The third-order Runge-Kutta method is used for the bubble center position and the Adams-Bashforth scheme for the radial equation (14).

A significant difference with respect to the method described in [35] is rooted in the bubble-related momentum and thermal source terms in the continuous-phase equations. These forcings are located at the center of each bubble and therefore, upon discretization of the equations, they have to be replaced with an equivalent system of forcings at the grid nodes. For this purpose, since in a staggered grid arrangement the momentum cells in the three directions are all different, force (6) exerted by a bubble is first decomposed into its radial, azimuthal, and vertical components. Each one of these components is then distributed by suitable weighing among the eight vertices of the surrounding momentum cell in the same direction. For example, for a radial force component $f$ at a position $r_{i}+\xi \Delta r, \theta_{j}+\eta \Delta \theta, z_{k}+\zeta \Delta z$, with $\Delta r$, $\Delta \theta$, and $\Delta z$, the grid spacings and $0 \leq \xi, \eta, \zeta<1$, the portion attributed to the node $\left(r_{i}, \theta_{j}, z_{k}\right)$ is

$$
(1-\xi)(1-\eta)(1-\zeta) f \text {. }
$$

The system of eight forces thus obtained produces the same net resultant and couple as the original bubble force. The same strategy has been used for the thermal forcing, so that the total amount of heat that each bubble exchanges with the liquid is preserved. It can be verified that this interpolation strategy is second-order accurate and therefore consistent with the overall spatial accuracy of the discretization.

The numerical solver has been validated by monitoring the temporal evolution of a single bubble in a quiescent flow without thermal effects. Furthermore, our results have been compared with the theoretical prediction of the lateral force induced on a spherical bubble rising with a constant velocity in a viscous fluid near a vertical cylindrical wall. We followed the theoretical method of Ref. [36] using the free-slip boundary condition for the bubble surface instead of the noslip condition used for a rigid particle. Another test of the numerical method and its implementation are offered by a comparison of the numerical results for the two sides of Eq. (29). Such a comparison is shown for a typical case in Fig. 3.

\section{IMPLEMENTATION}

From the numerical point of view, a significant practical difficulty of the present problem is the large difference between the flow time scale and the times over which bubbles grow and collapse. In order to have reasonable execution times of our computer code, it has been necessary to limit this difference by adopting a small cylinder size; we have taken a height $H=17.9 \mathrm{~mm}$ and a diameter $2 R=8.94 \mathrm{~mm}$. Furthermore, in order to limit the number of spatial cells necessary to resolve the flow, it is necessary to limit the Rayleigh number, which can be achieved by taking a small temperature difference; we take $T_{h}-T_{c}=0.25 \mathrm{~K}$. With these values and the physical properties of water at $373 \mathrm{~K}$, we have 
$\mathrm{Ra}=2 \times 10^{5}$. Since $T_{\text {sat }}=\frac{1}{2}\left(T_{h}+T_{c}\right)$, the hot plate is $0.125 \mathrm{~K}$ hotter than the saturation temperature, which in reality would not be a superheat sufficient to nucleate bubbles. This is another respect in which our model deviates from reality. On the other hand, since our focus here is the bubble effect on the thermal convection rather than the actual heat removal from the plate due to bubble formation, the compromise that is forced on us is less damaging than it might be in a study of boiling heat transfer. Assuming that the plate temperature is not significantly affected by the nucleation and the condensation of the bubbles is a reasonable idealization for small bubble numbers and highly thermally conductive boundaries.

The calculation is started without bubbles and run until the steady state shown in Fig. 1 is reached. At this point 25 - $\mu \mathrm{m}$-diameter bubbles are introduced randomly throughout the volume of the cylinder attributing to each one the local liquid velocity. Since, due to buoyancy, bubbles rise with respect to the liquid, they can reach the top plate with a nonzero velocity. When this happens, they are removed and replaced with new $25-\mu \mathrm{m}$-diameter bubbles centered at a random position at a height above the bottom plate equal to their radius; they are assigned the local liquid velocity. Bubbles reaching the lateral vertical wall of the cylinder are assumed to bounce elastically.

In order to avoid possible numerical problems due to the disappearance or excessive growth of bubbles, we have imposed artificial limits on the minimum and the maximum bubble diameters equal to 0.82 and $258 \mu \mathrm{m}$, respectively. We found however that these limits were never approached in our simulations. Since bubbles never condense completely, the total number of bubbles is constant in time.

\section{RESULTS}

Since bubbles tend to grow in volume in hotter liquid region, thus aiding buoyancy, and to condense in colder regions, they have a destabilizing effect on natural convection. These effects are clearly the stronger the larger the volume change. As explained before, in the present model, this feature can be controlled by controlling the Jakob number (3). A very small Jakob number may be thought of as a very large latent heat, which will tend to limit the volume change of the bubble, while, conversely, a large Jakob number would enhance the destabilizing effect.

While this is the major effect, there are other minor ones which operate in the opposite direction. For example, bubbles in a hot liquid region, for which $T>T_{\text {sat }}$, will tend to cool the liquid by absorbing heat and conversely in a colder liquid region. If $\mathrm{Ja}$ is very small, so that the bubble is prevented from growing appreciably, this process tends to eliminate the very temperature differences which drive the natural convection in the first place. With all other things being equal, the break-even point between increased buoyancy due to bubble expansion and decreased liquid buoyancy due to the bubble-induced cooling will be for that value of the Jakob number at which the thermal expansion of the bubble equals the added weight of the liquid due to the increased density. It will be seen from our results that this balance occurs for very small Ja so that, in most practical situations,

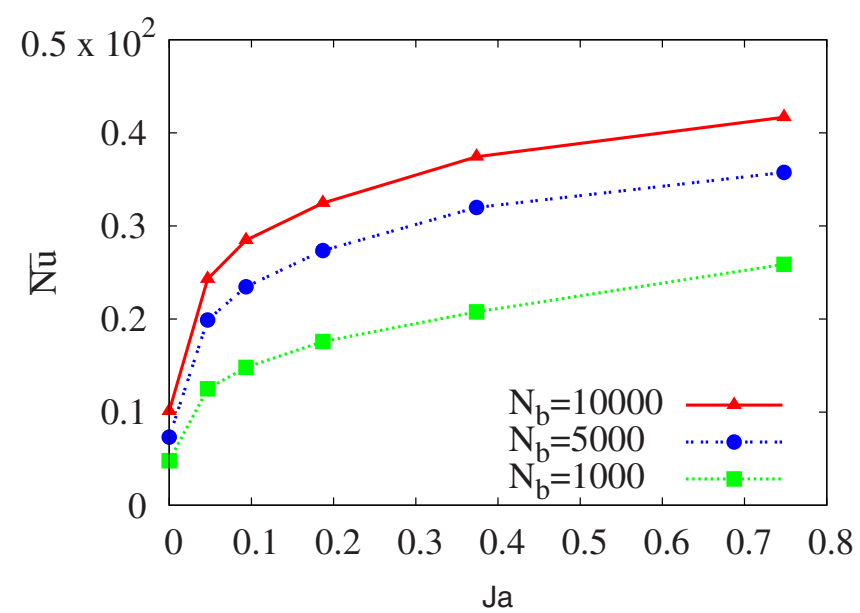

FIG. 4. (Color online) Averaged Nusselt number $\overline{\mathrm{Nu}}$ vs Jakob number for three different numbers of bubbles.

the balance will tip in favor of the enhanced buoyancy effect.

Figure 4 shows the effect on the average Nusselt number $\overline{\mathrm{Nu}}=\frac{1}{2}\left(\mathrm{Nu}_{h}+\mathrm{Nu}_{c}\right)$, defined in Eq. (30), of adding 1000, 5000, and 10000 bubbles to the basic single-phase RB flow; here, as in all the results shown, the Rayleigh number is $\mathrm{Ra}=2$ $\times 10^{5}$ and $\operatorname{Pr}=1.75$. Figure 5 shows the ratio of the bubble contribution

$$
\mathrm{Nu}_{\text {source }}=\frac{1}{\pi R^{2} k \Delta}\left\langle\sum_{i}\left(z_{i}-\frac{1}{2} H\right) Q_{i}\right\rangle_{t}
$$

to the average Nusselt number $\overline{\mathrm{Nu}}$. The remaining fraction of the Nusselt number is accounted for by conduction and pure convection, i.e., the first two terms in the right-hand sides of Eqs. (31) and (32). In both figures the horizontal axis is the Jakob number, which we use as a control parameter to investigate the effect of the added bubble buoyancy.

For $\mathrm{Ja}=0$, the bubbles maintain their initial diameter at injection at the plate $(25 \mu \mathrm{m})$ but, because they are kept at

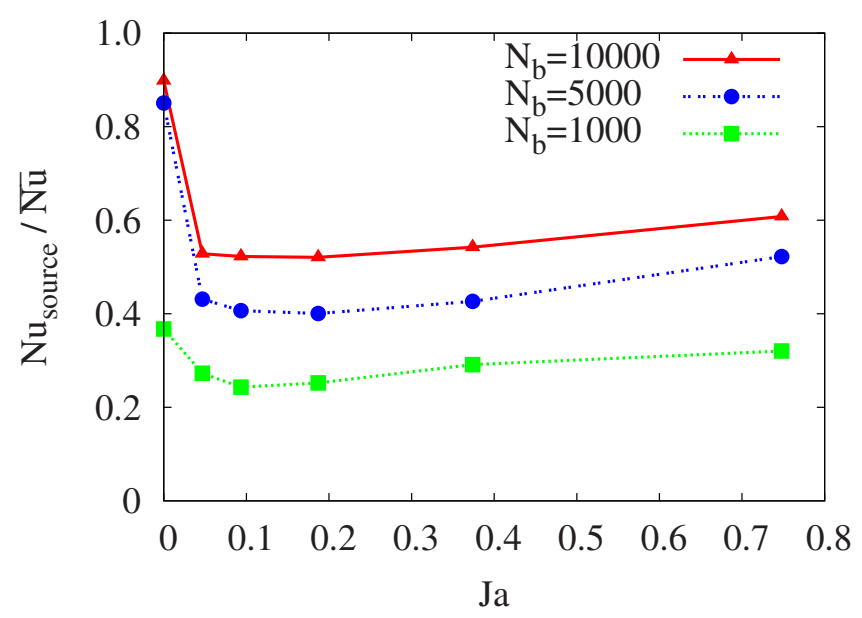

FIG. 5. (Color online) Ratio of the bubble source term (35) to the average Nusselt number (30). At small Ja, the additional bubbleinduced buoyant forcing is the dominant effect, while at large Ja the bubbles act as direct carriers of heat from the bottom to the top. 


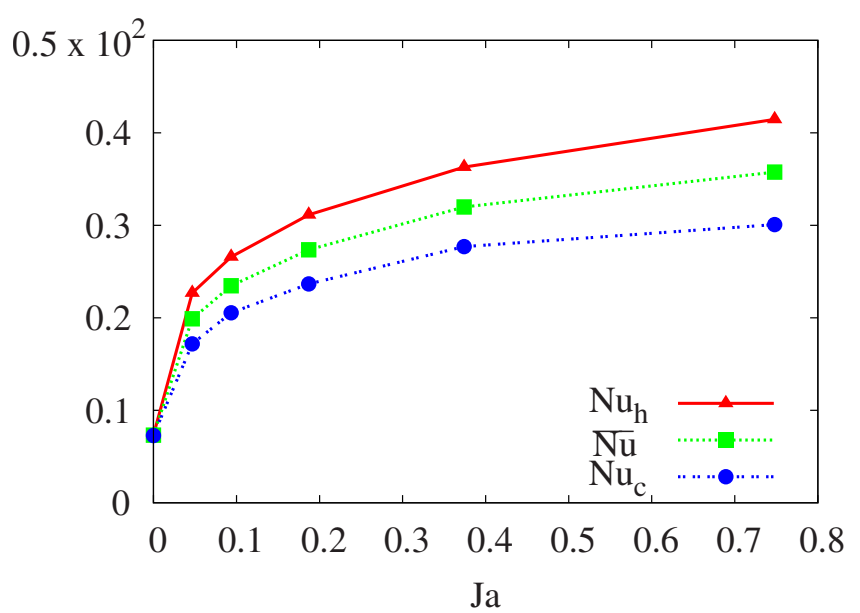

FIG. 6. (Color online) Comparison between the Nusselt numbers computed at the top and at the bottom boundaries for 5000 bubbles; the middle line is the average Nusselt number $\overline{\mathrm{Nu}}$ $=\frac{1}{2}\left(\mathrm{Nu}_{h}+\mathrm{Nu}_{c}\right)$. Here, we took $N_{b}=5000$.

$T_{\text {sat }}$, they cool the hotter liquid regions and heat up the cooler ones. As noted before, this behavior tends to stabilize the RB convection and is responsible for the fact that, while in the absence of bubbles the flow consists of an annular roll with an approximately horizontal axis (Fig. 1), the addition of $\mathrm{Ja}=0$ bubbles changes it to a toroidal roll with a vertical axis. Because of this stabilizing effect, the cooling and heating operated by the bubbles accounts for a large fraction of the total heat transported and, indeed, it can be seen from Fig. 5 that the bubble contribution (35) is very large, up to about $90 \%$ of the total for the 10000 bubble case. In principle, with enough bubble to stop the liquid circulation altogether, the contribution of the liquid to thermal transport would be reduced to simple conduction and the bubbles would give an even greater fraction of the total energy transported. Such a situation is purely hypothetical, however, as $\mathrm{Ja}=0$ is not realizable in practice.

As Ja is increased, the Nusselt number increases very rapidly at first (Fig. 4) due to the increased convection caused by buoyancy. As a consequence, the fraction of the total Nusselt number due to the bubbles (Fig. 5) undergoes a steep decline. With further increases in Ja, the Nusselt number keeps growing but at a more moderate rate. The minimum in the range $\mathrm{Ja}=0.1-0.2$ observed in Fig. 5 is due to a change in the flow structure as described later.

Figure 6 shows the Nusselt numbers computed at the top and the bottom of the cylinder and their average for 5000 bubbles; the behavior for the other bubble numbers is very similar. As shown by Eq. (29), the difference $\mathrm{Nu}_{c}-\mathrm{Nu}_{h}$ is due to the heat exchanged between the bubbles and the liquid. As the Jakob number begins to increase, the energy absorbed by each bubble per unit time increases because of a direct increase in the heat transfer coefficient of each individual bubble [see Eq. (20)] and an increase in the convective component of the bubble heat flux caused by the faster rise velocity of a larger bubble [Eq. (21)]. The moderation in the rate of growth of $\mathrm{Nu}$ at larger $\mathrm{Ja}$ is probably due to the increasing bubble rise velocity which limits their residence time in the cylinder.

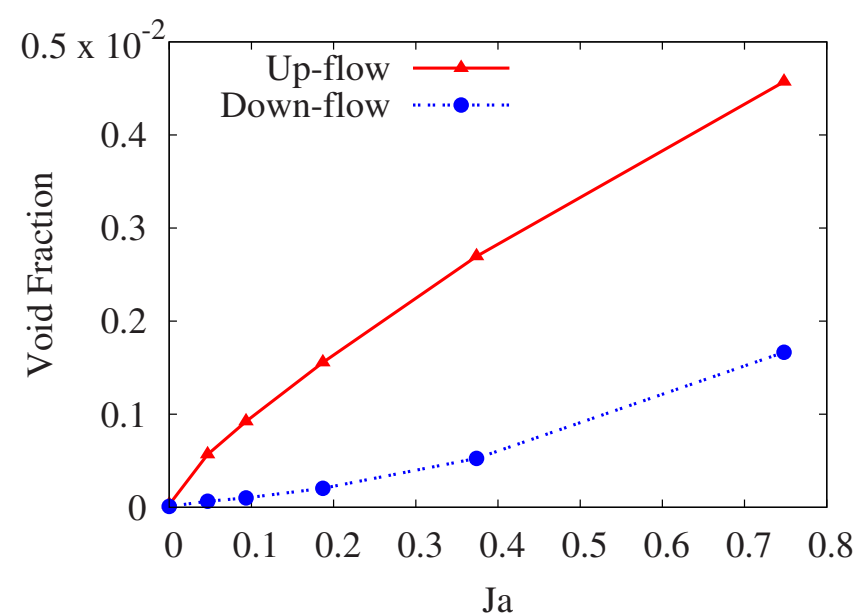

FIG. 7. (Color online) Average void fraction in the up-flow and in the down-flow regions for $N_{b}=5000$ bubbles.

By calculating the volume of bubbles located at regions of positive and negative vertical liquid velocities, we can look in detail at the effect of the increased buoyancy. Figure 7 shows the time- and the volume-averaged vapor volume fractions for 5000 bubbles as functions of the Jakob number. The results for the other cases are similar, with smaller void fractions for 1000 bubbles (for $\mathrm{Ja}=0.374$, approximately $0.015 \%$ and $0.092 \%$ ) and larger ones for 10000 bubbles (for $\mathrm{Ja}=0.374$, approximately $0.11 \%$ and $0.41 \%$ ). It is seen that the void fraction in the up-flow regions is consistently much larger than in the down-flow regions, thus providing strong evidence for the expected destabilizing effect of the buoyancy provided by the bubbles.

The void fraction reflects the combined effects of the bubble number and the bubble volume and it is interesting to consider these two contributions separately. The volume- and the time-averaged bubble radius $\left\langle R_{b}\right\rangle_{V, t}$ defined by

$$
\left\langle R_{b}\right\rangle_{V, t}=\left(\frac{3}{4 \pi N_{b}} \sum_{i}\left\langle V_{b i}\right\rangle_{t}\right)^{1 / 3}
$$

is shown in Fig. 8 as a function of the Jakob number for the case of Fig. 7 with 5000 bubbles. As expected, the bubble size increases markedly with the Jakob number and it tends to be somewhat larger in the hotter liquid regions. The timeand the volume-averaged fractions of the total bubble number in the up-flow and the down-flow regions, shown in Fig. 9 , indicates a strong tendency for bubbles to be in the hotter liquid regions, which is mostly responsible for the much larger void fraction in the rising liquid. This effect is probably due to fact that the newly injected bubbles at the hot plate tend to be swept up into the warm liquid by the convection current.

The results of Fig. 9 for the bubble numbers show that the difference between the fractions of bubbles in the up-flow and the down-flow regions is very large for small Jakob numbers and tends to decrease as Ja increases. This behavior can be understood looking at the change in the flow structure. 


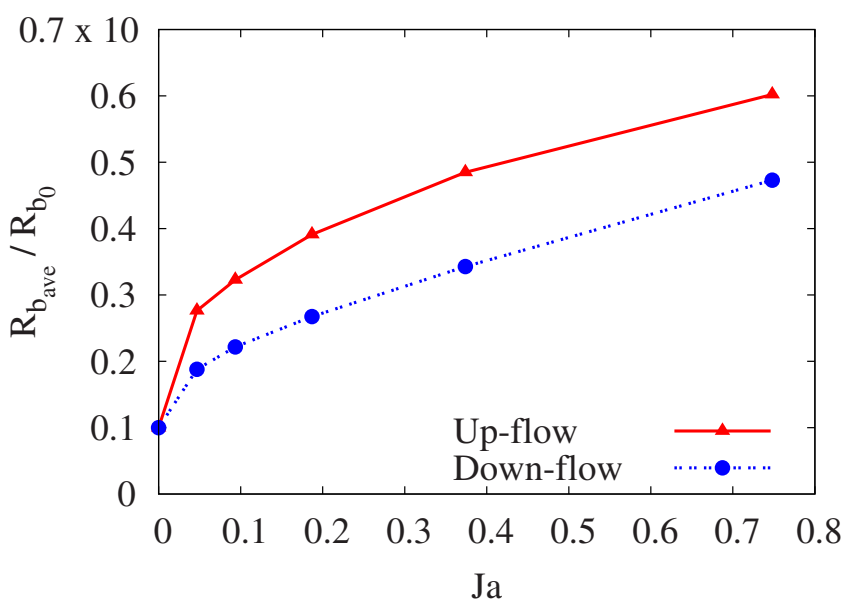

FIG. 8. (Color online) Averaged radius of the bubble computed in the up-flow and the down-flow regions for $N_{b}=5000$ bubbles; $R_{b 0}$ is the initial radius, $25 \mu \mathrm{m}$.

Without bubbles, the cylinder is occupied by a single convective roll which rises along one side and descends along the opposite side (Fig. 1). A picture of the flow for the 5000 bubbles, $\mathrm{Ja}=0$ case is shown in Fig. 10 where one vertical and three horizontal cross sections color- (or gray-) coded with the vertical velocity field are displayed. The blue structure in the proximity of the cylinder axis is the descending region of a toroidal vortex, while the remaining green areas are those where the liquid rises, mostly with a smaller velocity, except for a few faster zones (yellow and red). It can be seen here that the volume occupied by the rising liquid is much greater than that occupied by the descending liquid, and this circumstance offers a likely explanation of the much smaller fraction of bubbles in the latter noticeable in Fig. 9.

If the Jakob number is increased to $\mathrm{Ja}=0.0935$ (Fig. 11), the toroidal circulation is reinforced with a marked increase in the maximum rising and descending velocities (note that the color scales in these figures are not the same). For a still larger Jakob number, $\mathrm{Ja}=0.748$ (Fig. 12) the flow has changed back to a circulation rising along one side of the cylinder and descending along the opposite one, reminiscent

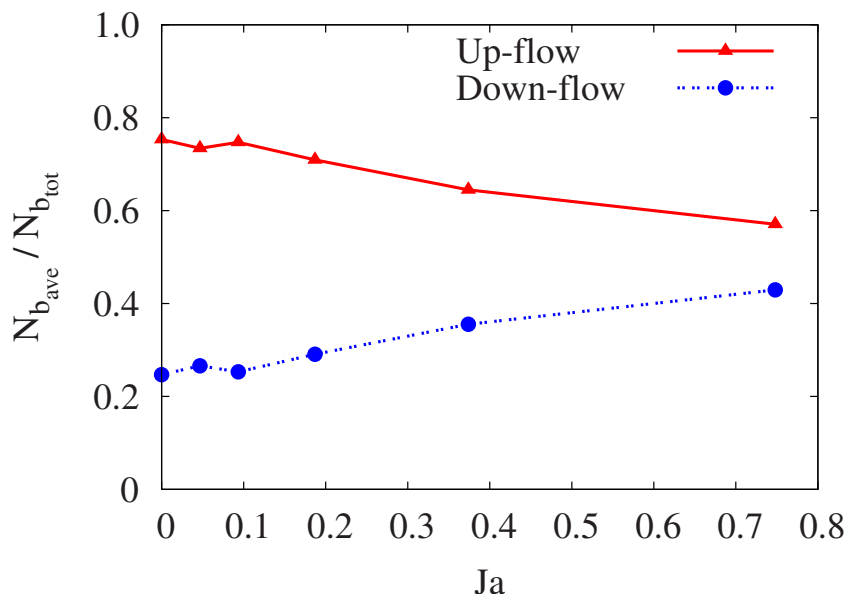

FIG. 9. (Color online) Averaged bubble numbers in the up-flow and the down-flow regions for $N_{b}=5000$ bubbles.

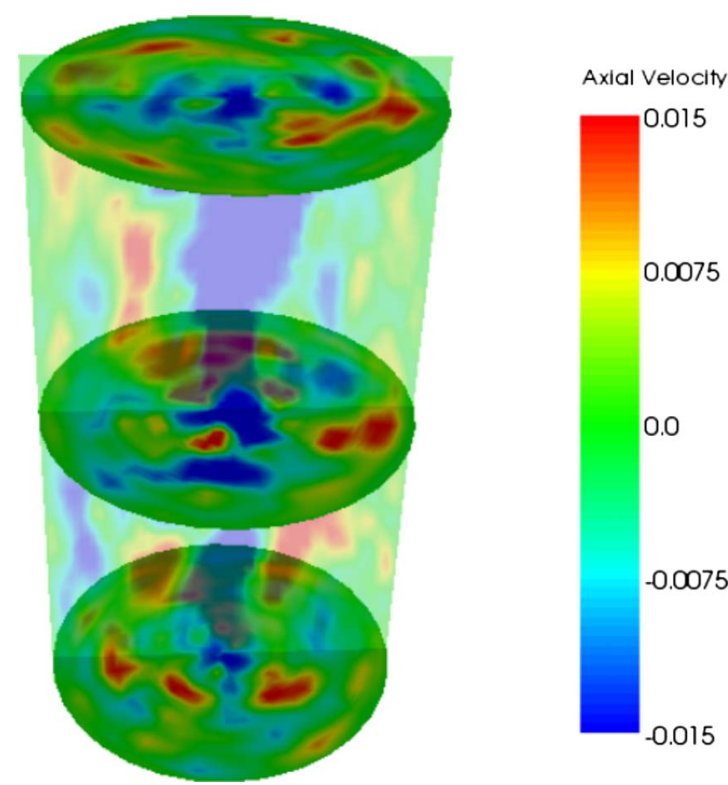

FIG. 10. (Color online) Vertical and horizontal cross sections (taken at $0.05 H, 0.5 H$, and $0.95 H$, respectively) of the vertical liquid velocity distribution in the cylinder for $\mathrm{Ja}=0$ and $N_{b}=5000$ bubbles. The blue (dark) structure near the axis is the descending region of the toroidal vortex which prevails for small Jakob numbers. The absolute values of the velocities are two orders of magnitude smaller as compared to the two subsequent figures as convection is suppressed at $\mathrm{Ja}=0$.

of the single-phase pattern of Fig. 1. Now the volumes occupied by the two streams are more balanced and the difference between the numbers of bubbles in the up-flow and the

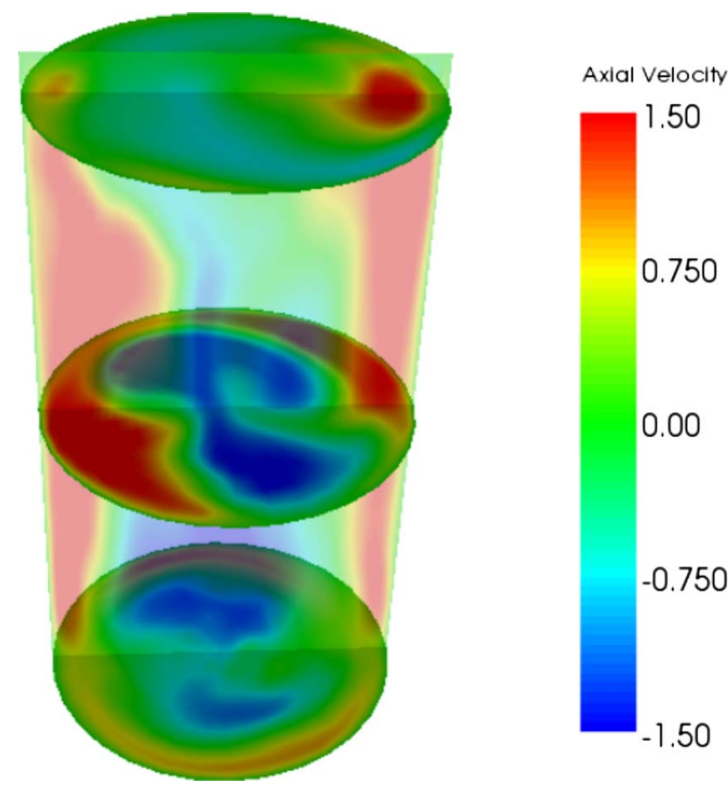

FIG. 11. (Color online) Vertical and horizontal cross sections (taken at $0.05 H, 0.5 H$, and $0.95 H$, respectively) of the vertical liquid velocity distribution in the cylinder for $\mathrm{Ja}=0.0935$ and 5000 bubbles. The blue (dark) structure near the axis is the descending region of the toroidal vortex which prevails for small Jakob numbers. 

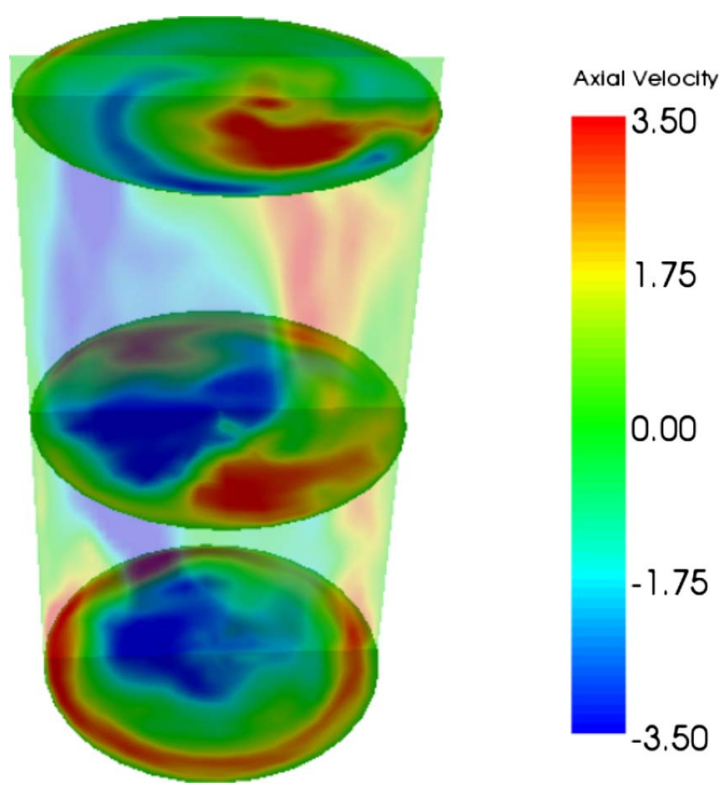

FIG. 12. (Color online) Vertical and horizontal cross sections (taken at $0.05 H, 0.5 H$, and $0.95 H$, respectively) of the vertical liquid velocity distribution in the cylinder for $\mathrm{Ja}=0.748$ and 5000 bubbles.

down-flow regions is smaller, as seen in Fig. 9, although the bubble fraction in the up flow is still larger than in the down flow.

These qualitative observations on the flow structure can be made quantitative by an analysis of the distribution of the liquid kinetic energy among different Fourier modes in the angular direction. We define the portion $E_{n}$ of the kinetic energy pertaining to mode $n$ by

$$
E_{n}=\frac{\pi}{\beta g H^{4} \Delta} \int_{0}^{R} r d r \int_{0}^{H} d z\left\langle\left|\mathbf{u}_{n}\right|^{2}\right\rangle_{t},
$$

where $\mathbf{u}_{n}$ is the $n$th Fourier coefficient (in the angular direction) of the vector velocity field. The mode $n=0$ is axisym-

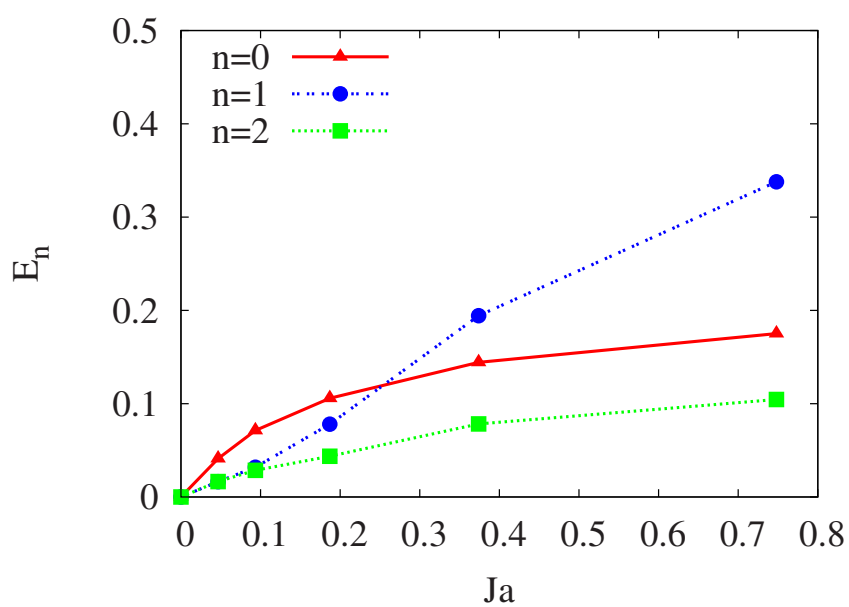

FIG. 13. (Color online) Fourier modes of the kinetic energy in the angular direction for $N_{b}=5000$ bubbles. Mode 0 corresponds to a toroidal vortex and mode 1 corresponds to a circulatory motion in the cell with approximately horizontal axis.

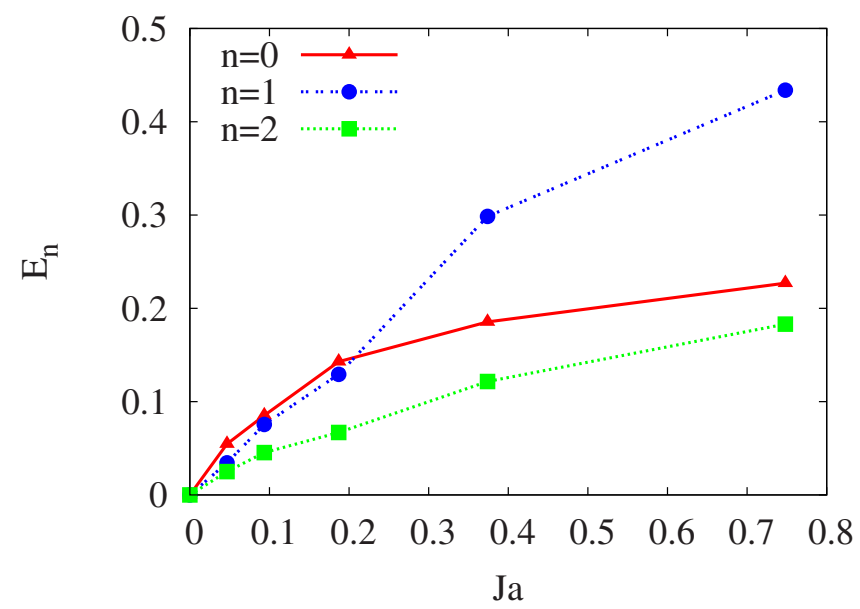

FIG. 14. (Color online) Fourier modes of the kinetic energy in the angular direction for $N_{b}=10000$ bubbles. Mode 0 corresponds to a toroidal vortex and mode 1 corresponds to a circulatory motion in the cell with approximately horizontal axis.

metric and corresponds to a toroidal circulation symmetric about the vertical axis of the cylinder; $n=1$ is a single vortex around an approximately horizontal axis, and the higher modes give further information on the details of the distribution of the flow over the cross section of the cylinder. Results for the $n=0,1$, and 2 modes are shown in Figs. 13 and 14 for 5000 and 10000 bubbles, respectively; the time averaging was carried out over the entire duration of the two-phase simulation. The values for $\mathrm{Ja}=0$ are very small, but nonzero. It is seen here that, for zero or small Jakob number, most of the kinetic energy is in the toroidal mode $n=0$. For larger values of $\mathrm{Ja}$, the energy in the $n=1$ mode rapidly increases giving rise to the flow structure exemplified in Fig. 12.

\section{SUMMARY AND CONCLUSIONS}

In this paper we have presented a simple model to simulate the effect of phase change and two-phase flow on natural convection. While, for the reasons given in Sec. V, the results must be considered as preliminary, we have found that the addition of bubbles has a profound effect on the flow structure and on the Nusselt number. Bubbles that are prevented from growing by artificially maintaining the Jakob number equal to zero (corresponding to an infinitely large latent heat of vaporization) tend to short-circuit temperature nonuniformities and to stabilize the convective motion. As the Jakob number is increased, the added buoyancy due to the bubble growth rapidly increases the circulation and the heat transport. As the Jakob number is increased further, the bubble growth becomes rapid, the residence time becomes short, and the rate of growth of the Nusselt number slows down. Correspondingly, with the increasing Jakob number, the structure of the convective flow in the cylinder undergoes significant changes.

\section{ACKNOWLEDGMENTS}

The authors express their gratitude to K. Sugiyama for numerous discussions and code validation calculations. L. E. 
Schmidt carried out some simulations on the finer grids. We moreover acknowledge SARA, Amsterdam, for supplying us with CPU time.

\section{APPENDIX A: ENTHALPY BALANCE EQUATIONS}

For a fluid medium, the microscopic form of the energy balance equation written in terms of the enthalpy per unit mass $h$ is

$$
\frac{\partial}{\partial t}(\rho h)+\nabla \cdot(\rho h \mathbf{u})-\frac{d p}{d t}=-\nabla \cdot \mathbf{q}
$$

in which $d p / d t=\partial p / \partial t+\mathbf{u} \cdot \nabla p$ and we have neglected the contribution due to viscous heating. Upon taking a volume average, this equation becomes (see, e.g., [37], p. 248)

$$
\begin{aligned}
& \frac{\partial}{\partial t}(\alpha\langle\rho h\rangle)+\nabla \cdot(\alpha\langle\rho h \mathbf{u}\rangle) \\
& \quad=\alpha\left\langle\frac{\partial p}{\partial t}+\mathbf{u} \cdot \nabla p\right\rangle-\nabla \cdot(\alpha\langle\mathbf{q}\rangle)-\frac{1}{\mathcal{V}} \int_{S_{i}}(\dot{m} h+\mathbf{q}) \cdot \mathbf{n} d S_{i},
\end{aligned}
$$

where $\alpha$ is the volume fraction, $\dot{m}$ is the mass flux, the angular brackets indicate averages, and the integral in the last term is over the interfaces contained in the averaging volume. In applying this relation to the vapor phase, we set $\alpha$ $=n v$, with $v$ as the bubble volume, and note that then $\alpha\langle\rho h\rangle=n H_{b}$, with $H_{b}=v\left\langle\rho_{V} h_{V}\right\rangle$ as the mean enthalpy per bubble. The temperature and pressure are very nearly uniform and constant inside the bubbles and $\alpha$ is very small, so that we can neglect the terms with the heat flux $\mathbf{q}$ in Eq. (A2). Upon setting

$$
\frac{1}{\mathcal{V}} \int_{S_{i}} \dot{m} h_{V} d S_{i}=\sum_{i} Q_{i} \delta\left(\mathbf{x}-\mathbf{x}_{i}\right),
$$

we recover Eq. (9). Note that here we approximate the vapor enthalpy by the latent heat, which is permissible since the liquid enthalpy is much smaller than that of the vapor in the temperature range of concern here.

For the liquid, it is more convenient to start from the enthalpy equation written in terms of the temperature, namely,

$$
\rho c_{p} \frac{D T}{D t}=-\nabla \cdot \mathbf{q}_{L},
$$

which already includes the assumption of incompressibility. Upon averaging and assuming that the liquid volume fraction is very close to 1 , we find the first three terms of Eq. (7) plus an interfacial contribution. The simplest way to evaluate the latter is to note that, at the interface, the enthalpy exchanges must balance each other so that the interfacial contribution in the liquid equation must be the negative of that shown in Eq. (A3).

\section{APPENDIX B: EXACT RELATIONS FOR THE KINETIC AND THE THERMAL DISSIPATIONS $\epsilon_{u}$ AND $\epsilon_{\theta}$}

Upon multiplying the momentum equation (5) by $\mathbf{u}$ and averaging over the cylinder volume and time, we find, by the no-slip condition on the cylinder walls,

$$
\epsilon_{u} \equiv \nu\left\langle\partial_{j} u_{i} \partial_{j} u_{i}\right\rangle_{V}=\beta g\left\langle\left(T-T_{s a t}\right) u_{3}\right\rangle_{V}+\frac{1}{\rho V} \sum_{i}\left\langle\mathbf{f}_{i} \cdot \mathbf{u}\right\rangle_{t} .
$$

The term $\left\langle\left(T-T_{s a t}\right) u_{3}\right\rangle_{V}$ can be eliminated in terms of the single-phase Nusselt number at the hot base of the cylinder, given by Eq. (31), to find

$$
\begin{aligned}
\epsilon_{u}= & \frac{\nu^{3}}{H^{4}} \frac{\mathrm{Ra}}{\operatorname{Pr}^{2}}\left(\mathrm{Nu}_{h}-1\right)+\frac{1}{\rho V} \sum_{i}\left\langle\mathbf{f}_{i} \cdot \mathbf{u}\right\rangle_{t} \\
& -\frac{\beta g}{\rho c_{p} V}\left\langle\sum_{i}\left(z_{i}-H\right) Q_{i}\right\rangle_{t}
\end{aligned}
$$

in which $V=\pi R^{2} H$ is the volume of the cylinder. Alternatively, in terms of the Nusselt number at the cold top of the cylinder,

$$
\epsilon_{u}=\frac{\nu^{3}}{H^{4}} \frac{\mathrm{Ra}}{\operatorname{Pr}^{2}}\left(\mathrm{Nu}_{c}-1\right)+\frac{1}{\rho V} \sum_{i}\left\langle\mathbf{f}_{i} \cdot \mathbf{u}\right\rangle_{t}-\frac{\beta g}{\rho c_{p} V}\left\langle\sum_{i} z_{i} Q_{i}\right\rangle_{t} .
$$

The thermal dissipation $\epsilon_{\theta}$ is defined in terms of

$$
\theta=T-\frac{1}{2}\left(T_{h}+T_{c}\right)=T-T_{\text {sat }}
$$

as $\epsilon_{\theta}=\kappa\left\langle|\nabla \theta|^{2}\right\rangle_{V, t}$. An expression for this quantity may be readily obtained by multiplying the energy equation by $\theta$ and averaging over the cylinder volume and time to find

$\epsilon_{\theta}=\frac{\kappa\left(T_{h}-T_{c}\right)}{2 H}\left[-\left\langle\partial_{3} T\right\rangle_{A, t, z=H}-\left\langle\partial_{3} T\right\rangle_{A, t, z=0}\right]+\frac{1}{\rho c_{p}}\left\langle\sum_{i} \theta_{i} Q_{i}\right\rangle_{t}$,

where we have used the assumed insulation of the lateral walls and the fact that $\theta= \pm \frac{1}{2}\left(T_{h}-T_{c}\right)$ at the bottom and the top of the cylinder. The temperature gradients can be eliminated in terms of the Nusselt numbers $\mathrm{Nu}_{h, c}$ to find

$$
\epsilon_{\theta}=\frac{\kappa \Delta^{2}}{H^{2}} \frac{\mathrm{Nu}_{h}+\mathrm{Nu}_{c}}{2}+\frac{1}{\rho c_{p} V}\left\langle\sum_{i}\left(T_{i}-T_{s a t}\right) Q_{i}\right\rangle_{t},
$$

which replaces the well-known relation $\epsilon_{\theta}=\left(\kappa \Delta^{2} / H^{2}\right) \mathrm{Nu}$ of the single-phase RB convection. 
[1] L. P. Kadanoff, Phys. Today 54(8), 34 (2001).

[2] G. Ahlers, S. Grossmann, and D. Lohse, Rev. Mod. Phys. 81, 503 (2009).

[3] V. Dhir, Annu. Rev. Fluid Mech. 30, 365 (1998).

[4] J. Q. Zhong, D. Funfschilling, and G. Ahlers, Phys. Rev. Lett. 102, 124501 (2009).

[5] B. Bunner and G. Tryggvason, J. Fluid Mech. 466, 17 (2002).

[6] B. Bunner and G. Tryggvason, J. Fluid Mech. 466, 53 (2002).

[7] A. Esmaeeli and G. Tryggvason, Phys. Fluids 17, 093303 (2005).

[8] A. Mukherjee and V. Dhir, ASME Trans. J. Heat Transfer 126, 1023 (2004).

[9] S. Elghobashi and G. C. Truesdell, J. Fluid Mech. 242, 655 (1992).

[10] M. Boivin, O. Simonin, and K. Squires, J. Fluid Mech. 375, 235 (1998).

[11] A. Ferrante and S. Elghobashi, Phys. Fluids 15, 315 (2003).

[12] E. Climent and J. Magnaudet, Phys. Rev. Lett. 82, 4827 (1999).

[13] I. Mazzitelli, D. Lohse, and F. Toschi, Phys. Fluids 15, L5 (2003).

[14] I. Mazzitelli, D. Lohse, and F. Toschi, J. Fluid Mech. 488, 283 (2003).

[15] K. Sugiyama, E. Calzavarini, and D. Lohse, J. Fluid Mech. 608, 21 (2008).

[16] D. Legendre, J. Borée, and J. Magnaudet, Phys. Fluids 10, 1256 (1998).

[17] O. E. Ivashnyov and N. N. Smirnov, Phys. Fluids 16, 809 (2004).

[18] M. R. Maxey, E. Chang, and L. Wang, Exp. Therm. Fluid Sci.
12, 417 (1996).

[19] B. Yang and A. Prosperetti, J. Fluid Mech. 601, 253 (2008).

[20] J. Magnaudet and I. Eames, Annu. Rev. Fluid Mech. 32, 659 (2000).

[21] E. A. van Nierop et al., J. Fluid Mech. 571, 439 (2007).

[22] R. Mei, J. F. Klausner, and C. J. Lawrence, Phys. Fluids 6, 418 (1994).

[23] D. Legendre and J. Magnaudet, J. Fluid Mech. 368, 81 (1998).

[24] J. Magnaudet and D. Legendre, Phys. Fluids 10, 550 (1998).

[25] G. K. Batchelor, An Introduction to Fluid Dynamics (Cambridge University Press, Cambridge, England, 1967).

[26] J. Magnaudet, M. Rivero, and J. Fabre, J. Fluid Mech. 284, 97 (1995).

[27] T. R. Auton, J. Fluid Mech. 183, 199 (1987).

[28] M. Rivero, J. Magnaudet, and J. Fabre, C. R. Acad. Sci. Paris II 312, 1499 (1991).

[29] E. J. Chang and M. R. Maxey, J. Fluid Mech. 303, 133 (1995).

[30] R. Mei and J. Klausner, Phys. Fluids A 4, 63 (1992).

[31] D. A. Labuntsov, B. A. Kolchygin, E. A. Zacharova, and L. N. Vladimirova, Teplofiz. Vys. Temp. 2, 446 (1964).

[32] E. Ruckenstein, Chem. Eng. Sci. 10, 22 (1959).

[33] R. Verzicco and P. Orlandi, J. Comput. Phys. 123, 402 (1996).

[34] P. N. Swarztrauber, SIAM (Soc. Ind. Appl. Math.) J. Numer. Anal. 11, 1136 (1974).

[35] R. Verzicco and R. Camussi, J. Fluid Mech. 477, 19 (2003).

[36] M. Shinohara and H. Hashimoto, J. Phys. Soc. Jpn. 46, 320 (1979).

[37] Computational Methods in Multiphase Flow, edited by A. Prosperetti and G. Tryggvason (Cambridge University Press, Cambridge, UK, 2007). 